\title{
On fractionally integrated logistic smooth transitions in time series
}

\author{
Olanrewaju I.Shittu' ${ }^{1}$, OlaOluwa S. Yaya ${ }^{2}$ \\ Department of Statistics University of Ibadan, Nigeria \\ Email: ${ }^{1}$ oi.shittu@mail.ui.edu.ng, ${ }^{2}$ os.yaya@mail.ui.edu.ng
}

\begin{abstract}
Long memory and nonlinearity are two key features of some macroeconomic time series which are characterized by persistent shocks that seem to rise faster during recession than it falls during expansion. A variant of nonlinear time series model (STAR) together with long cation memory is proposed to examine these features in inflation series for three economies. This paper therefore seeks to examine the dynamics and application of FILSTAR model on inflation rates with a view to obtaining better parameter estimates. The results which compares favourably with that of Van Dijk, et al. (2002) elicit some interesting attributes on inflation in the developed and developing economies.
\end{abstract}

Keywords: Fractional integration, Long memory, Smooth transition autoregression, Inflation rates, RSTAR, Time series.

JEL Classification: C22, C51, C87.

\section{INTRODUCTION}

Many real world problems do not satisfy the assumptions of linearity and/or stationarity. Thus, the era of nonlinear modelling has come to replace linear modelling in financial or econometric time series. Also, classical theory of stationarity and linearity may not apply to some economic, finance and macroeconomic series because they consider series at their levels, $I(0)$; first order integration, $I(1)$ as well as higher order of integration (Box and Jenkins, 1976). Hassler and Wolters (1995) considered the fractional integration where $I(0<d<0.5)$ for inflation data from five industrial countries and found that the series are all within the long memory range.

The nonlinearity property of economic series can be justified by the existence of asymmetry in the series. In order to consider these possible nonlinearities, it is necessary to have econometric models that are able to generate different dynamics according to the business cycle phase. (see Granger and Teräsvirta (1993); Teräsvirta (1994)). Van Dijk et al. (2002) present the modelling cycle for specification of STAR model which include estimation of differencing parameter, testing for nonlinearity, parameter estimation and model adequacy tests, in the case where the transition function is the logistic function and applied this on US monthly unemployment rate. Smallwood (2005) and Boutahar et al. (2008) extend these results to the fractionally integrated STAR model with an exponential transition function. The model was applied to measure the purchasing power by considering the real exchange rate data for twenty countries. This model is still new and has to be tried beyond its applicability to exchange rates.

This paper therefore seeks to examine the dynamics and application of FILSTAR model on inflation rates with a view to obtaining better parameter estimates and reliable forecasts. The remaining sections of the paper are then organized as follows: Section 2 gives the general review of the FISTAR model and the linearity tests. Section 3 discusses the estimation of the model; Section 4 presents the results of the analysis and Section 5 gives the conclusion.

THE FISTAR MODEL SPECIFICATION

A Fractionally Integrated (FI) time series process $\left\{X_{t}\right\}, t=1, \ldots, T$ is considered as

$(1-B)^{d} X_{t}=y_{t}$

where $B$ is the backward shift operator, $d$ is the noninteger fractional differenced parameter and $y_{t}$ is a covariance-stationary process. For fractionally integrated process in (1), the integration parameter $d$ assumes values within the stationary and invertible range $-0.5<d<0.5$ (Sowell, 1992a; Mayoral, 2007). For $0<d<0.5, X_{t}$ is a stationary long memory process in the sense that autocorrelations are not 
absolutely summable but rather at a much slower hyperbolic rate. It exhibits nonstationary process if $0.5 \leq d<1$.

Applying the Maclaurin's series expansion around $B=0$, the fractional difference operator is expanded as,

$$
(1-B)^{d}=1-d B-\frac{d(1-d) B^{2}}{2 !}-\ldots=\sum_{j=0}^{\infty} \frac{\Gamma(-d+j)}{\Gamma(-d) \Gamma(j+1)} B^{j}
$$

where the Euler gamma function,

$$
\Gamma(z)=\int_{0}^{\infty} s^{z-1} e^{-s} d z=(z-1) ! \text {. }
$$

Based on (2) and (1), the fractionally integrated STAR (FISTAR) model of order $p$ is expressed as,

$$
\begin{gathered}
\sum_{j=0}^{\infty} \frac{\Gamma(-d+j)}{\Gamma(-d) \Gamma(j+1)} B^{j} X_{t}=y_{t} \\
y_{t}=\phi_{1}^{\prime} \tilde{y}_{t}^{(p)}\left(1-F\left(s_{t} ; \gamma, c\right)\right)+\phi_{2}^{\prime} \tilde{y}_{t}^{(p)} F\left(s_{t} ; \gamma, c\right)+\varepsilon_{t} \\
\text { where } \quad t=1,2, \ldots, T, \quad \tilde{y}_{t}^{(p)}=\left(1, y_{t-1}, \ldots, y_{t-p}\right)^{\prime},
\end{gathered}
$$$$
\phi_{i}=\left(\phi_{i 0}, \phi_{i 1}, \ldots, \phi_{i p}\right)^{\prime} \text { and } i=1,2 \text {. The } \varepsilon_{t} \text { is assumed to }
$$
be a difference sequence distributed with $E\left(\varepsilon_{t} \mid \Omega_{t-1}\right)=0 \quad$ and $\quad E\left(\varepsilon_{t}^{2} \mid \Omega_{t-1}\right)=\sigma^{2} \quad$ with $\Omega_{t-1}=y_{t-1}, y_{t-2}, \ldots, y_{1-(p-1)}, y_{1-p}$ representing the past history of the time series. Following Teräsvirta (1994), the transition variable $S_{t}$ is assumed to be a lagged endogenous variable, that is, $s_{t}=y_{t-l}$ for certain integer $0<l \leq p$. At point $l$, nonlinearity is sharper. For the case where 1 or 2 autoregressive parameters determine the linear part of the STAR model, the inequality $l>p$ holds. The transition function, $F\left(s_{t} ; \gamma, c\right)$ is either of logistic or exponential form (Teräsvirta, 1994):

$$
\begin{aligned}
& F\left(s_{t} ; \gamma, c\right)=\frac{1}{1+\exp \left(-\gamma\left(s_{t}-c\right)\right)}, \quad \gamma>0 \\
& F\left(s_{t} ; \gamma, c\right)=1-\exp \left(-\gamma\left(s_{t}-c\right)^{2}\right), \quad \gamma>0
\end{aligned}
$$

The $\gamma$ is the slope parameter and $c$, the intercept in the transition function. From the model above, it is clear that the model reduces to linear ARFI $(p)$ models when $F\left(s_{t} ; \gamma, c\right)=0$ or 1 that is shifting between two extreme linear regimes after staying in nonlinear region for some time. The fractional parameter $d$, the autoregressive parameters, $\phi_{i}$ and nonlinear parameters, $\gamma$ and $c$ make the FISTAR model potentially useful for capturing both long memory and nonlinear smooth transition features of the time series, $X_{t}$ (Boutahar et al., 2008).

STAR modelling approach of Teräsvirta (1994) has been modified to capture our specification procedure for FISTAR, as it is proposed by van Dijk et al. (2002):

1. Specify a linear $\operatorname{ARFI}(p)$ model by selecting the autoregressive order $p$ by means of Akaike and Schwarz information criteria (Akaike,1974 and Schwarz, 1978).

2. Test the null hypothesis of linearity against the alternative of a FISTAR model.

3. Specify the model STAR model by choosing between the two competing transition functions.

4. Estimate the parameters in the specified FISTAR model.

5. Evaluate the estimated model using misspecification tests (no residual autocorrelation, serial correlation, normality test, ARCH test and others).

Teräsvirta (1994) follows the approach of Luukkonen, Saikkonen and Teräsvirta (1988) in replacing the transition function $F\left(s_{t} ; \gamma, c\right)$ with a suitable Taylor series approximation about $\gamma=0$ and test linearity by means of a Lagrange multiplier (LM) statistic. Luukkonen, Saikkonen and Teräsvirta (1988) then consider testing the null hypothesis $H_{0}: \gamma=0$ of linearity against the alternative of LSTAR nonlinearity by using the LSTAR function. The third order Taylor's series approximation of the logistic model is then given as auxiliary regression model,

$y_{t}=\phi^{\prime} \tilde{y}_{t}^{(p)}+\beta_{1}^{\prime} \tilde{y}_{t}^{(p)} s_{t}+\beta_{2}^{\prime} \tilde{y}_{t}^{(p)} s_{t}^{2}+\beta_{3}^{\prime} \tilde{y}_{t}^{(p)} s_{t}^{3}+\tilde{\varepsilon}_{t}$

where $\beta_{i}=\left(\beta_{i 1}, \ldots, \beta_{i p}\right)^{\prime}, i=1,2,3$ are functions of the parameters $\phi_{1}, \phi_{2}, \gamma$ and $c$. The null hypothesis then becomes $H_{0}: \beta_{1}=\beta_{2}=\beta_{3}=0$, which implies the selection of linear model. The approach of Teräsvirta (1994) is to specify the model based on the nested hypotheses:

$H_{O 1}: \beta_{3}=0$

$H_{O 2}: \beta_{2}=0 \mid \beta_{3}=0$

$H_{O 3}: \beta_{1}=0 \mid \beta_{2}=\beta_{3}=0$ 
which is supported by Escribano and Jordá (2001). This sequence of hypotheses implies that rejection of $H_{O 1}$ suggests acceptance of LSTAR model. Also, rejection of $\mathrm{H}_{\mathrm{O} 2}$ is an acceptance of ESTAR model. Lastly, rejection of $\mathrm{H}_{\mathrm{O}}$ implies the selection of LSTAR model. Analytically, the test procedures follow:

1. Regressing $y_{t}$ on $\left\{1, y_{t-j} ; j=1,2, \ldots, p\right\}$ to form $\hat{\varepsilon}_{t},(t=1,2, \ldots, T)$ and computing the residual sum of squares $S S R_{0}=\sum_{t=1}^{T} \hat{\varepsilon}_{t}^{2}$;

2. regressing

$$
\hat{\varepsilon}_{t}
$$

on

$\left\{1, y_{t-i}, y_{t-i} s_{t}^{k}, ; i=1,2, \ldots, p ; k=1,2,3\right\}$

to

form the residuals $\widetilde{\varepsilon}_{t}(t=1,2, \ldots, T)$ and $S S R_{1}=\sum_{t=1}^{T} \tilde{\varepsilon}_{t}^{2}$ and

3. computing the

$F=\frac{\left(S S R_{0}-S S R_{1}\right) / 3(p+1)}{S S R_{0} /(N-4(p+1))}$ which is $\chi^{2}$ distributed with $3(p+1)$ degree of freedom.

\section{ESTIMATION OF FISTAR PARAMETERS}

The estimation of FISTAR model starts by estimating the fractional difference parameter in the series using the rescaled statistics of Hurst (1951). The rescaled statistic (R/S) defined as:

$R / S=1 / S_{T}(q)\left(\sup _{1 \leq m \leq T} \sum_{j=1}^{m}\left(X_{j}-\bar{X}\right)-\inf _{1 \leq m \leq T} \sum_{j=1}^{m}\left(X_{j}-\bar{X}\right)\right)$

where $S_{T}$ is the MLE estimate of standard deviation from time series, $X_{j}$. Then, $S_{T}(q)=S_{T}+2 \sum_{j=1}^{q} w_{j}(q) \hat{\gamma}_{j}$ and

$w_{j}(q)=1-j /(q+1)$ such that $q<T$ (Lo, 1991). The Hurst coefficient, $H$ is then estimated by,

$$
\hat{H}=\frac{1}{\log (T)} \log (R / S) .
$$

The fractional differencing parameter, $d$ is then obtained as,

$$
\hat{d}=\hat{H}-0.5 \text {. }
$$

The approximate values of $y_{t}$ can be obtained in the time domain as in Sowell $(1992 a, b)$. The time domain approach follows the Binomial Theorem representation of $(1-B)^{d}$. This implies that $y_{t}$ is approximated by using $\hat{d}$ estimated by the Hurst estimation approach and a truncated fractionally differenced series is given as,

$$
\begin{aligned}
\tilde{y}_{t} & =\sum_{j=t}^{\infty} \frac{\Gamma(-\hat{d}+j)}{\Gamma(-\hat{d}) \Gamma(j+1)} X_{t-j} \\
& =\sum_{k=0}^{\infty} \frac{\Gamma(-\hat{d}+t+k)}{\Gamma(-\hat{d}) \Gamma(t+k+1)} X_{k}
\end{aligned}
$$

From (12), it is clear to set $X_{t-j}=0$ for $t-j$ outside of the sample, $T$.

The second transformation approach uses the frequency domain approach of Geweke and PorterHudak (1983). Here, the Fourier transform of the observed series, $X_{t}$ is pre-multiplied by the Fourier transform of the fractional differencing operator based on $\hat{d}$, and then compute the inverse Fourier transform. The final series obtain follows ARMA $(p, q)$ process.

According to van Dijk et. al. (2002), after the estimation of the fractional difference parameter, all the remaining parameters in the STAR model are estimated together. Beran (1995) suggests approximate maximum likelihood (AML) estimator for invertible and possible nonstationary ARFIMA models which allows for regime switching autoregressive dynamics. This estimator then minimizes the sum of squared residual of the FISTAR model as,

$S\left(\phi_{1}, \phi_{2}, \gamma, c\right)=\sum_{i=1}^{T} \varepsilon_{t}^{2}\left(\phi_{1}, \phi_{2}, \gamma, c\right)$.

The estimation principle can be performed using any optimization procedure. We now consider the choice of appropriate starting value parameters and the estimation of the smoothness parameter in the transition function. The estimation procedure can be simplified by concentrating the sum of squares function since the parameters $\gamma$ and $c$ in the transition function imply STAR model of parameters $\phi_{1}$ and $\phi_{2}$ and this makes the FISTAR model linear in the remaining parameters (Leybourne, Newbold and Vougas (1998), 
van Dijk et al. (2002)). Then, estimates of $\phi=\left(\phi_{1}^{\prime}, \phi_{2}^{\prime}\right)^{\prime}$ is obtained by ordinary least squares (OLS) as

$$
\hat{\phi}(\gamma, c)=\frac{\sum_{t=1}^{T} \tilde{y}_{t}^{(p)}(\gamma, c) y_{t}}{\sum_{t=1}^{T} \tilde{y}_{t}^{(p)}(\gamma, c) \tilde{y}_{t}^{(p)}(\gamma, c)^{\prime}}
$$

where

$$
\tilde{y}_{t}^{(p)}(\gamma, c)=\left[\tilde{y}_{t}^{\prime(p)}\left(1-F\left(s_{t} ; \gamma, c\right), \tilde{y}_{t}^{\prime(p)} F\left(s_{t} ; \gamma, c\right)\right]^{\prime}\right.
$$

and the notation $\hat{\phi}(\gamma, c)$ is used to indicate that the estimate of $\phi$ is conditional upon $\gamma$ and $c$. Thus, the sum of squares function $Q_{N}(\varphi)$ can be concentrated with respect to $\phi_{1}$ and $\phi_{2}$ as,

$$
Q_{N}(\gamma, c)=\sum_{t=1}^{T}\left[y_{t}-\hat{\phi}(\gamma, c)^{\prime} \tilde{y}_{t}^{(p)}(\gamma, c)\right]^{2}
$$

and $Q_{N}(\gamma, c)$ will be minimized with respect to parameters $\gamma$ and $c$ only. The estimate of $\gamma$ is very difficult to obtain when it is large because its large value makes the STAR model to be similar to threshold autoregressive (TAR) model as the transition function, $F\left(s_{t} ; \gamma, c\right)$ comes close to a step function and this function is then standardized. To obtain an accurate estimate, there should be many observations, $s_{t}$ in the neighbourhood of $c$ and this implies small deviation. Sensible starting value for the nonlinear optimization of the STAR model can easily be obtained by considering a two dimensional grid search over $\gamma$ and $c$. Then for any set of the three values $\left(A_{j}^{c}, A_{k}^{\gamma}\right)$, the parameter vectors $\left(\phi_{1}, \phi_{2}\right)$ are then estimated through ordinary least squares (OLS). The outcome of this is a set of estimates, $\quad\left(\hat{\phi}_{1}^{0}, \hat{\phi}_{2}^{0}, \hat{\gamma}^{0}, \hat{c}^{0}\right)$. Practically, most estimation software for STAR modelling are designed to follow the specification, $y_{t}=\phi_{1}^{\prime} \tilde{y}_{t}^{(p)}+\left(\phi_{2}-\phi_{1}\right)^{\prime} \tilde{y}_{t}^{(p)} F\left(s_{t} ; \gamma, c\right)+\varepsilon_{t}$

where the nonlinear part is on one side of the model.

\section{DATA ANALYSIS AND RESULTS}

The monthly data on inflation are sourced variously from Federal Reserve Bank of St. Louis (US Inflation), National Bureau of Statistics (Nigerian Inflation) and Office of National Statistics (UK Inflationa). These series range from January 1991 to December 2009 ( $T$ $=228$ ). Preliminary analyses is performed using EViews 5 software from Quantitative Micro Software, LLC and Smooth Transition Regression (STR) analysis is performed by the RSTAR contributed package developed for the analysis of nonlinear time series (Balcilar, 2008), made available through $\mathrm{R}$ Development Core Team (2009).

Table 1: Descriptive Statistics on Time Series

\begin{tabular}{l|c|c|c}
\hline Statistics & $\begin{array}{c}\text { Nigeria Inflation } \\
\text { Series }\end{array}$ & $\begin{array}{c}\text { US Inflation } \\
\text { Series }\end{array}$ & $\begin{array}{c}\text { UK Inflation } \\
\text { Series }\end{array}$ \\
\hline \hline Minimum & 0.90 & -2.10 & 0.50 \\
Maximum & 78.50 & 5.65 & 8.50 \\
Mean & 22.020 & 2.650 & 2.351 \\
Std. Dev. & 19.856 & 1.179 & 1.574 \\
Skewness & 1.362 & -0.867 & 2.252 \\
Kurtosis & 3.573 & 5.855 & 8.063 \\
Jarque- & & & \\
Bera & 73.596 & 105.957 & 436.189 \\
Probability & 0.0000 & 0.0000 & 0.0000 \\
\hline
\end{tabular}

From Table 1, the mean inflation rate during the period under study is 22.020 for Nigeria, 2.65 for US and 2.35 for the UK with standard deviation of 19.856, 1.178 and 1.574 respectively. The three series show the assymetric and leptokurtic nature of inflation series in the three economies as indicated by the level of skewness and Jarque-Bera test.

In Figure 1, the plot of the inflation series for the three economies shows the great disparity in the inflation series of Nigeria as compared with that of the US and the UK. 


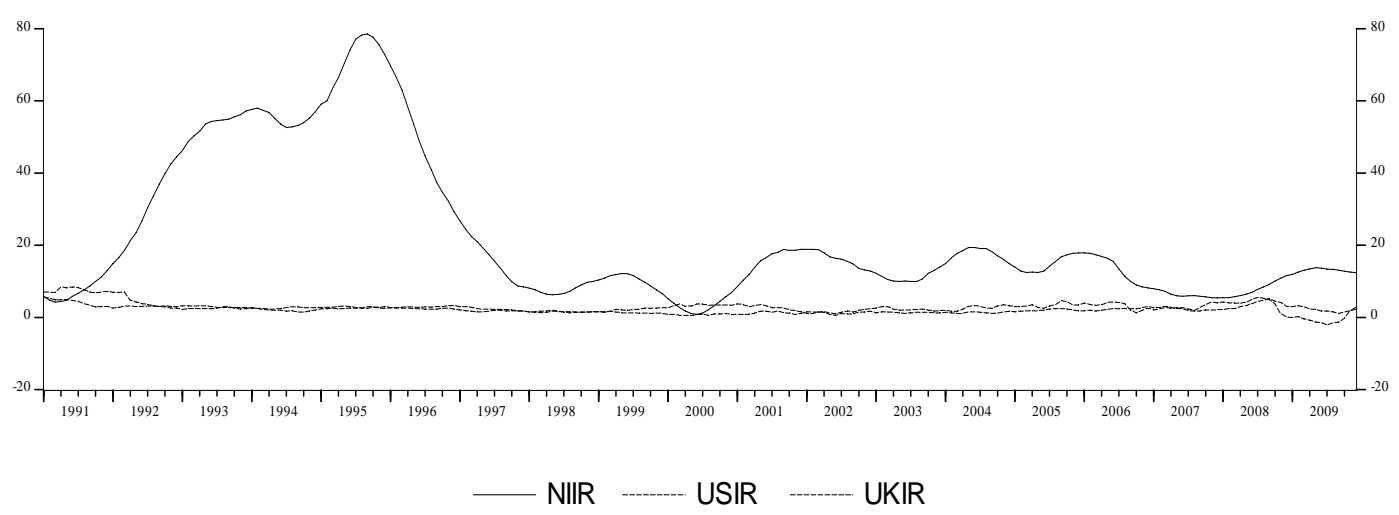

Fig. 1: Time Plots of Inflation Rates

In order to examine stationarity in the time series, the augmented Dickey Fuller (ADF) unit root test was carried out under the null hypothesis of unit root. The results as given in Table 2 indicate rejection of the null hypothesis of unit root (series nonstationary) at 1 , 5 and $10 \%$ for Nigeria, US and UK inflation series. The series are further subjected to KPSS (Kwiatkowski, Phillips, Schmidt and Shin) test of 1992. The null hypothesis is rejected at $5 \%$. This is an evidence of long memory.

Having noticed the presence of long memory, the differencing parameter was obtained using the Hurst (1951) method. The results are shown in Table 3. The observed series were differenced again using the estimated values $(0 \leq d \leq 0.5)$ and re-examined for stationarity and the result is shown in Table 4.
Modelling cycle of FISTAR model continues by fitting linear AR models to the inflation series. Optimal models were obtained based on minimum values of AIC and SIC. So, AR (2), AR (4) and AR (2) models are optimal models for Nigeria, US and UK inflation series respectively. Table 5 shows the result of the first stage in nonlinear STAR testing. Nonlinearity is found to be sharper at different lags, $l=4, l=3$ and $l=1$ for Nigerian, US and UK inflation series. These are determined as least significant points for $0<l \leq p$ or $l>p$. Note that this is determined based on $l>p$, that is certain point outside the model lags. Nonlinear smooth transition are tested in these sharper points. The test results as given in Table 6 shows at least the significance of one of the $\beta_{i}$ based on the auxiliary regression in (7) which is an indication that the three inflation series exhibit nonlinear STAR behaviour.

Table 2: Stationarity Tests on Inflation Time Series

\begin{tabular}{|c|c|c|c|c|c|c|c|c|c|}
\hline & \multicolumn{3}{|c|}{ Nigerian Inflation Series } & \multicolumn{3}{|c|}{ US Inflation Series } & \multicolumn{3}{|c|}{ UK Inflation Series } \\
\hline Test & \multicolumn{2}{|c|}{ ADF } & KPSS & \multicolumn{2}{|c|}{ ADF } & KPSS & \multicolumn{2}{|c|}{ ADF } & KPSS \\
\hline Statistic & \multicolumn{2}{|c|}{-3.522} & 0.752 & \multicolumn{2}{|c|}{-4.589} & 0.737 & \multicolumn{2}{|c|}{-3.055} & 1.212 \\
\hline $1 \%$ & -3.459 & & 0.739 & -3.459 & & 0.739 & -3.459 & & 0.739 \\
\hline $5 \%$ & -2.874 & $(0.0083)$ & 0.463 & -2.874 & $(0.0002)$ & 0.463 & -2.874 & $(0.0083)$ & 0.463 \\
\hline $10 \%$ & -2.574 & & 0.347 & -2.574 & & 0.347 & -2.574 & & 0.347 \\
\hline
\end{tabular}


Am. J. Sci. Ind. Res., 2010, 1(3): 439-447

Table 3: Estimation of Fractional Difference Parameters

\begin{tabular}{l|l|l|l}
\hline & \multicolumn{3}{|c}{ Non-Parametric Approach } \\
\hline & Nigerian Inflation Series & US Inflation Series & UK Inflation Series \\
\hline \hline$\hat{d}$ & 0.3289 & 0.1542 & 0.2819 \\
$R / S$ & $(90.0325)$ & $(34.8870)$ & $(69.7733)$ \\
\hline
\end{tabular}

Table 4: Stationarity Tests on Fractionally Differenced Time Series

\begin{tabular}{|c|c|c|c|c|c|c|c|c|c|}
\hline & \multicolumn{3}{|c|}{ Nigerian Inflation Series } & \multicolumn{3}{|c|}{ US Inflation Series } & \multicolumn{3}{|c|}{ UK Inflation Series } \\
\hline Test & \multicolumn{2}{|c|}{ ADF } & KPSS & \multicolumn{2}{|c|}{ ADF } & KPSS & \multicolumn{2}{|c|}{ ADF } & KPSS \\
\hline Statistic & \multicolumn{2}{|c|}{-3.122} & 0.416 & \multicolumn{2}{|c|}{-3.186} & 0.1639 & \multicolumn{2}{|c|}{-6.046} & 0.2543 \\
\hline $1 \%$ & -3.459 & & 0.739 & -3.459 & & 0.739 & -3.459 & & 0.739 \\
\hline $5 \%$ & -2.874 & $(0.0264)$ & 0.463 & -2.874 & $(0.0222)$ & 0.463 & -2.874 & $(0.0000)$ & 0.463 \\
\hline $10 \%$ & -2.574 & & 0.347 & -2.574 & & 0.347 & -2.574 & & 0.347 \\
\hline
\end{tabular}

Table 5: Determination of the Transition Variable, $s_{t}=y_{t-l}$

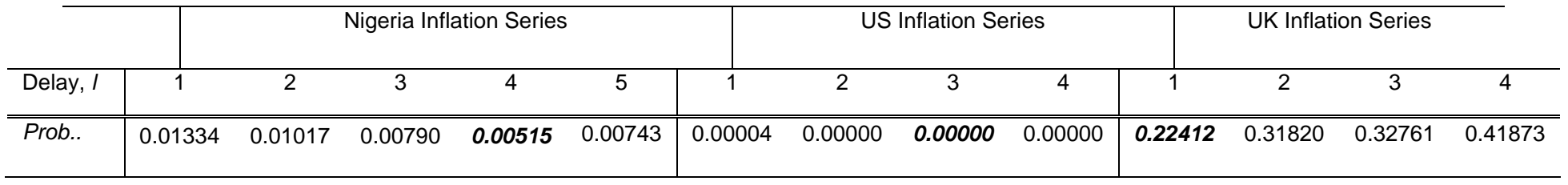

Based on the nested hypothesis in (8), LSTAR models are specified for the three inflation rates unlike ESTAR model specified for exchange rates in (Boutahar, 2008).

Table 6: STAR Nonlinearity Test and Model Specification

\begin{tabular}{l|ccc|ccc|ccc}
\hline & \multicolumn{3}{|c|}{ Nigeria Inflation Series } & \multicolumn{3}{c|}{ US Inflation Series } & \multicolumn{3}{c}{ UK Inflation Series } \\
\hline$\beta_{i}$ & 1 & 2 & 3 & 1 & 2 & 3 & 1 & 2 & 3 \\
\hline \hline Prob.. & 0.37617 & $\mathbf{0 . 0 0 0 9 4 2 1 6}$ & 0.43241 & $\mathbf{0 . 0 3 1 4 3 8}$ & $\mathbf{8 . 3 4 E - 0 7}$ & $\mathbf{0 . 0 0 0 1 9}$ & 0.60882 & 0.69793 & $\mathbf{0 . 0 3 6 0 7}$ \\
\hline Model & \multicolumn{3}{|c|}{ LSTAR } & & & LSTAR & & & LSTAR \\
\hline
\end{tabular}

Estimation results are presented in Tables 7-9. Note subset of the insignificant nonlinear parameters cannot be taken in the RSTAR software. So, the STAR and FISTAR models presented are optimal. 
Am. J. Sci. Ind. Res., 2010, 1(3): 439-447

Table 7: Estimated LSTAR Model for Nigerian Inflation Rates

\begin{tabular}{|c|c|c|c|c|c|c|c|c|c|}
\hline & Model & \multicolumn{2}{|c|}{ AR } & \multicolumn{2}{|c|}{ ARFI } & \multicolumn{2}{|c|}{ LSTAR } & \multicolumn{2}{|c|}{ FILSTAR } \\
\hline & Estimator & Estimates & Prob. & Estimates & Prob. & Estimates & Prob. & Estimates & Prob. \\
\hline \multirow{4}{*}{ Linear part } & $\overline{\hat{\phi}_{10}}$ & 21.65910 & 0.00000 & 1.64978 & 0.0000 & 0.23993 & 0.0001 & 0.03211 & 0.4513 \\
\hline & $\hat{\phi}_{11}$ & 1.93548 & 0.00000 & -0.48204 & 0.0001 & 1.98177 & 0.0000 & 1.58115 & 0.0000 \\
\hline & $\hat{\phi}$ & -0.94128 & 0.00005 & -0.18114 & 0.0030 & -1.00173 & 0.0000 & -0.32336 & 0.0538 \\
\hline & $\hat{\phi}_{13}$ & & & & & & & -0.26088 & 0.0035 \\
\hline \multirow[t]{8}{*}{ Nonlinear part } & $\hat{\phi}_{20}$ & & & & & 2.79471 & 0.0398 & 1.65748 & 0.2963 \\
\hline & $\hat{\phi}_{1}$ & & & & & -0.03051 & 0.0309 & -0.64939 & 0.0815 \\
\hline & $\hat{\phi}_{2}$ & & & & & & & 0.17342 & 0.7273 \\
\hline & $\hat{\phi}_{23}$ & & & & & & & 0.95191 & 0.0324 \\
\hline & $\hat{\phi}_{24}$ & & & & & & & -0.67563 & 0.0014 \\
\hline & $\hat{\gamma}$ & & & & & 2.92593 & 0.1887 & 10.98377 & 0.3251 \\
\hline & $\hat{C}$ & & & & & 43.88512 & 0.0000 & 15.10189 & 0.0000 \\
\hline & $\hat{l}$ & & & & & 4 & & 4 & \\
\hline \multirow{4}{*}{ Diagnostic tests } & $A I C$ & 332.0028 & & 1.3771 & & 300.2108 & & 299.1587 & \\
\hline & SIC & 342.2908 & & 1.4226 & & 324.0610 & & 336.6868 & \\
\hline & $R^{2}$ & 0.9994 & & 0.9959 & & 0.9995 & & 0.9963 & \\
\hline & $A R C H-L M$ & 0.5657 & 0.4528 & 1.8344 & 0.1770 & 2.08314 & 0.1504 & 3.43958 & 0.0650 \\
\hline
\end{tabular}

In Table 7, the slope parameter for LSTAR is $\hat{\gamma}=2.925$ and for FISTAR is $\hat{\gamma}=10.983$. The above shows that the transition rate between one regime and the other is slow for LSTAR and faster in FISTAR model as indicated by the magnitude of the parameter estimates. Here, fractional integration does not improve the model fit but the FI series still retained nonlinearity property. Since the series are confirmed to have long memory, the difference parameter had to be estimated. This implies that ARFI and FISTAR models are preferred to AR and STAR models respectively (see Van Dijk et al., 2002).

Table 8 also shows the estimated slope parameters of $\hat{\gamma}=7.677$ for LSTAR and $\hat{\gamma}=6.057$ for FISTAR models indicating that the transition between the two regimes of STAR models is slow. The values of the slope parameters are closed to each other because of small values of the difference parameters, $(\hat{d}=0$ for LSTAR and $\hat{d}=0.1542$ for FISTAR).

From Table 9, value of the slope parameter dropped from $\hat{\gamma}=33.7800$ to $\hat{\gamma}=6.05677$ indicating fast to slow smooth transitioning from one regime to the other. 
Table 8: Estimated LSTAR Model for US Inflation Rates

\begin{tabular}{|c|c|c|c|c|c|c|c|c|c|}
\hline & Model & \multicolumn{2}{|c|}{ AR } & \multicolumn{2}{|c|}{ ARFI } & \multicolumn{2}{|c|}{ LSTAR } & \multicolumn{2}{|c|}{ FILSTAR } \\
\hline & Estimator & Estimates & Prob. & Estimates & Prob. & Estimates & Prob. & Estimates & Prob. \\
\hline \multirow{5}{*}{ Linear part } & $\hat{\phi}_{10}$ & 2.72725 & 0.0000 & 1.24780 & 0.0000 & 0.16843 & 0.1370 & 0.01088 & 0.6068 \\
\hline & $\hat{\phi}_{11}$ & 1.43073 & 0.0000 & -0.54730 & 0.0000 & 1.28605 & 0.0000 & 1.16477 & 0.0000 \\
\hline & $\hat{\phi}_{12}$ & -0.73275 & 0.0000 & 0.17335 & 0.0113 & -0.52379 & 0.0000 & -0.49817 & 0.0000 \\
\hline & $\hat{\phi}_{13}$ & 0.36304 & 0.0024 & & & 0.17673 & 0.0239 & 0.24095 & 0.0084 \\
\hline & $\hat{\phi}_{14}$ & -0.14600 & 0.0397 & & & & & & \\
\hline \multirow{6}{*}{ Nonlinear part } & $\hat{\phi}_{20}$ & & & & & 5.36344 & 0.0818 & -1.67135 & 0.3084 \\
\hline & $\hat{\phi}_{21}$ & & & & & 1.13672 & 0.0443 & 0.86859 & 0.3403 \\
\hline & $\hat{\phi}_{22}$ & & & & & -2.24124 & 0.0123 & & \\
\hline & $\hat{\gamma}$ & & & & & 7.67672 & 0.5270 & 6.05677 & 0.4182 \\
\hline & $\hat{C}$ & & & & & 4.90000 & 0.0000 & 1.64402 & 0.0000 \\
\hline & $\hat{l}$ & & & & & 3 & & 3 & \\
\hline \multirow{4}{*}{ Diagnostic tests } & $A / C$ & 206.8093 & & 0.8805 & & 179.6015 & & 186.8999 & \\
\hline & SIC & 223.9560 & & 0.9260 & & 210.3063 & & 214.2287 & \\
\hline & $R^{2}$ & 0.8953 & & 0.8223 & & 0.9067 & & 0.8383 & \\
\hline & $A R C H-L M$ & 1.9750 & 0.1187 & 1.5736 & 0.1967 & 2.1640 & 0.1426 & 4.3252 & 0.0377 \\
\hline
\end{tabular}

.Table 9: Estimated LSTAR Model for UK Inflation Rates

\begin{tabular}{|c|c|c|c|c|c|c|c|c|c|}
\hline & Model & \multicolumn{2}{|c|}{ AR } & \multicolumn{2}{|c|}{ ARFI } & \multicolumn{2}{|c|}{ LSTAR } & \multicolumn{2}{|c|}{ FILSTAR } \\
\hline & Estimator & Estimates & Prob. & Estimates & Prob. & Estimates & Prob. & Estimates & Prob. \\
\hline \multirow{4}{*}{ Linear part } & $\hat{\phi}_{10}$ & 3.285163 & 0.0065 & \multirow{4}{*}{0.84805} & \multirow{4}{*}{0.0000} & \multirow{4}{*}{$\begin{array}{r}0.133100 \\
1.06600 \\
-0.13540\end{array}$} & \multirow{2}{*}{$\begin{array}{l}0.0164 \\
0.0000\end{array}$} & 0.01088 & 0.6068 \\
\hline & $\hat{\phi}_{11}$ & 1.12114 & 0.0000 & & & & & 1.16477 & 0.0000 \\
\hline & $\hat{\phi}_{12}$ & \multirow{2}{*}{-0.13552} & \multirow[t]{2}{*}{0.0443} & & & & 0.0354 & -0.49817 & 0.0000 \\
\hline & $\hat{\phi}_{13}$ & & & & & & & 0.24095 & 0.0084 \\
\hline \multirow[t]{5}{*}{ Nonlinear part } & $\hat{\phi}_{20}$ & & & & & 1.59100 & 0.5418 & -1.67135 & 0.3084 \\
\hline & $\hat{\phi}_{21}$ & & & & & -0.184300 & 0.5709 & 0.86859 & 0.3403 \\
\hline & $\hat{\gamma}$ & & & & & 33.7800 & 1.0000 & 6.05677 & 0.4182 \\
\hline & $\hat{c}$ & & & & & 6.02500 & 1.0000 & 1.64402 & 0.0000 \\
\hline & $\hat{l}$ & & & & & 1 & & 1 & \\
\hline \multirow{4}{*}{ Diagnostic tests } & $A / C$ & 137.9201 & & 0.5497 & & 129.4520 & & 186.8999 & \\
\hline & SIC & 148.2081 & & 0.7789 & & 153.3958 & & 214.2287 & \\
\hline & $R^{2}$ & 0.9566 & & 0.8335 & & 0.9574 & & 0.8383 & \\
\hline & $A R C H-L M$ & 0.0003 & 0.9856 & 0.0059 & 0.9391 & 0.05279 & 0.8185 & 1.70649 & 0.1931 \\
\hline
\end{tabular}




\section{CONCLUSION}

In this paper, we have considered model which captures persistence and nonlinearities in time series data. The dynamics and application of FILSTAR model on inflation rates were examined with a view to obtaining better parameter estimates. Long memory, as indicated in the dynamics of inflation was combined with a nonlinear model that is capable of identifying market structure in two phases: recession and expansion. Detection of long memory in the nonlinear series did not improve the fit of the model as indicated by the diagnostic tests. It is interesting to note that removal of long memory in a series does not lead to removal of nonlinearity. Meanwhile, the results show some interesting atrributes of inflation in the developed and developing economies, and compare favourably with US unemployment model of Van Dijk, et al. (2002).

\section{REFERENCES}

Akaike, H. (1974). "A new look at the statistical model identification". IEEE Transactions on Automatic Control AC 19: 716-723.

Balcilar, M. (2008). RSTAR: Analysis of nonlinear time series. $\quad \mathrm{R}$ package version 0.1-1. http://www.mbalcilar.net, http://www.r-project.org

Beran, J. (1995). "Maximum likelihood estimation of the differencing parameter for invertible short and long memory autoregressive integrated moving average models", Journal of the Royal Statistical Society, Series B, 57 (4): 659-672.

Box, G. E. P. and Jenkins, G. M. (1976). Time Series Analysis: Forecasting and Control,

2nd ed. Holden-Day: San Francisco.

Boutahar, M. et. al. (2008). "A Fractional Integrated Exponential STAR Model Applied to the US Effective Exchange Rates", GREQAM, pp. 1-27.

Escribano, Á. and Jordá, O. (2001). "Testing nonlinearity: Decision Rules for Selecting between Logistic and Exponential STAR models". Spanish Economic review. 3: $193-209$

EViews (2004). EViews 5 User's Guide. Quantitative Micro Software, LLC, U.S.A

Granger, C.W.J. and Teräsvirta, T. (1993). Modelling Nonlinear Economic Relationships. Oxford: Oxford University Press. Chinese edition 2006: Shanghai University of Finance \& Economics Press.

Hassler, U. and Wolters, J. (1995). "Long memory in Inflation Rates: International Evidence". Journal of Business \& Economic Statistics, 13 (1): 37-45.
Hurst, H. (1951). "Long term Storage capacity of Reservoirs". Transactions of the American Society of Civil Engineers, 116:770-779.

Kwiatkowski, D. Phillips, P.C.B., Schmidt, P. and Shin, Y. (1992). "Testing the Null Hypothesis of Stationary against the Alternative of a Unit Root," Journal of Econometrics, 54, 159-178.

Leybourne, S., Newbold, P. and Vougas, D. (1998). "Unit roots and smooth transitions," Journal of Time Series Analysis, 19: 55-62.

Lo, A.W. (1991). "Long term memory in Stock market prices", Econometrica 59: 1279-1313.

Luukkonen, R. Saikkonen, P. and T. Teräsvirta (1988). "Testing Linearity against Smooth Transition Autoregressive models". Biometrika 75: 491-499.

Mayoral, L. (2007): "Minimum Distance Estimation of Stationary and non-Stationary ARFIMA Processes", Journal of Econometrics, 10: 124-148.

R Development Core Team (2009). R: A language and environment for statistical computing. R Foundation for Statistical Computing, Vienna, Austria. ISBN 3-90005107-0, URL http://www.R-project.org.

Schwarz, G. (1978). "Estimating the dimension of a model". Annals of Statistics, 6: 461-464.

Smallwood, A. D. (2005)."Joint tests for non-linearity and long memory: the case of purchasing power parity", Studies in Nonlinear Dynamics and Econometrics 9(2): 1-28.

Sowell, F. (1992a). "Maximum Likelihood Estimation of Stationary univariate Fractionally Integrated time Series Models," Journal of Econometrics 53: 165-188.

Sowell, F. (1992b). "Modelling Long run behaviour with Fractional ARIMA model". Journal of Monetary Economics, 29: 277-302.

Teräsvirta, T. (1994). "Specification, Estimation and Evaluation of Smooth Transition Autoregressive Models". Journal of the American Statistical Association, 89: 208-218.

Van Dijk, D., Franses, P. H. and Paap, R. (2002). "A nonlinear Long Memory model, with an Application to US Unemployment". Journal of Econometrics, 110: 135-165 